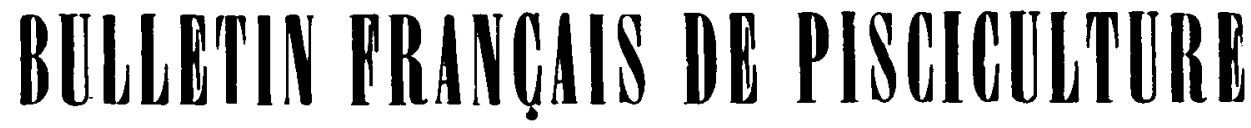

\section{INFLUENCE DE LA CASÉINE IODÉE ET DE LA THYROXINE SUR LA CROISSANCE DE LA JEUNE TRUITE ARC-EN-CIEL (Salmo gairdneri).}

Par M. FONTAINE et M. M. BARADUC (Laboratoire de Physiologie de l'Institut Océanographique).

Nous avons été souvent frappés de la diversité des régimes proposés aux jeunes Salmonidés dans les piscicultures, et en particulier des différences importantes qui existent entre les teneurs de ces régimes en iode, certains pisciculteurs nourrissant leurs poissons avec de la viande provenant de l'abattage du bétail, d'autres avec du poisson de mer. Ces régimes diffèrent certainement non seulement par leur teneur en iode minéral, mais aussi par leur teneur en iode engagé dans des substances de nature protéique ou protido-lipidique.

Or, parmi celles-ci, il en est dont l'intervention sur la croissance du bétail a été bien mise en évidence et il nous a paru intéressant d'en rechercher l'action éventuelle sur la croissance de la Truite arc-en-ciel.

Ces Truites provenaient de piscicultures de la région parisienne et étaient gardées dans des aquariums à eau courante de l'Institut Océanographique. Ces aquariums étaient situés dans un sous-sol normalement obscur, mais qui était éclairé au moins trois heures par jour par des lampes "lumière du jour".

Le régime de base était de la viande de boucherie (muscle de bœuf généralement), aliment pauvre en iode $(0,0035 \mathrm{mg}$. d'iode pour 100 grammes d'après les tables de composition des aliments de L. RaNDOIN).

L'iode était ajouté soit sous forme de thyroxine (produit cristallisé Hoffman la Roche), soit sous forme de caséine iodée (produit que la maison BYLA nous a procuré gracieusement, ce dont nous la remercions vivement). Les Truites étaient divisées en trois lots.

I. - Aliment de base + une dose déterminée de caséine égale à celle des deux lots suivants.

II. - Aliment de base + la dose précitée de caséine, mais ici sous forme de caséine iodée. 
III. - Aliment de base + la dose précitée de caséine pure + une dose de thyroxine correspondant (par son action sur le métabolisme respiratoire du Rat) à l'activité physiologique de la dose de caséine iodée du lot II.

Une différence à souligner entre les régimes des lots II et III réside donc dans le fait que, pour une activité hormonale comparable (d'après l'action sur le métabolisme respiratoire du Rat), le lot II est beaucoup plus riche en iode que le lot III.

Les Truites ètaient nourries une fois par jour, tous les jours sauf le dimanche.

Nous donnerons quelques exemples des résultats obtenus.

Expérience $n^{\circ}$ 1. - Trois lots de 20 Truites de poids moyen de 24 grammes, suivies du 17 Novembre 1951 au 1 er Février 1952. Température de l'eau variant de 5 à $12^{\circ}$. La caséine iodée (à $7 \%$ d'iode) titrait $1,2 \%$ en thyroxine. La caséine du lot III était donc additionnée de 1,2\% de thyroxine. Les quantités quotidiennes de caséine ajoutées au régime de base furent pour chaque Truite de :

$40 \mathrm{mg}$. du 17 Novembre au 10 Décembre,

$50 \mathrm{mg}$. du 10 Décembre au 7 Janvier,

$60 \mathrm{mg}$. du 7 Janvier au $1^{\text {er Février. }}$

La courbe ci-dessous (fig. I) rassemble les résultats obtenus.

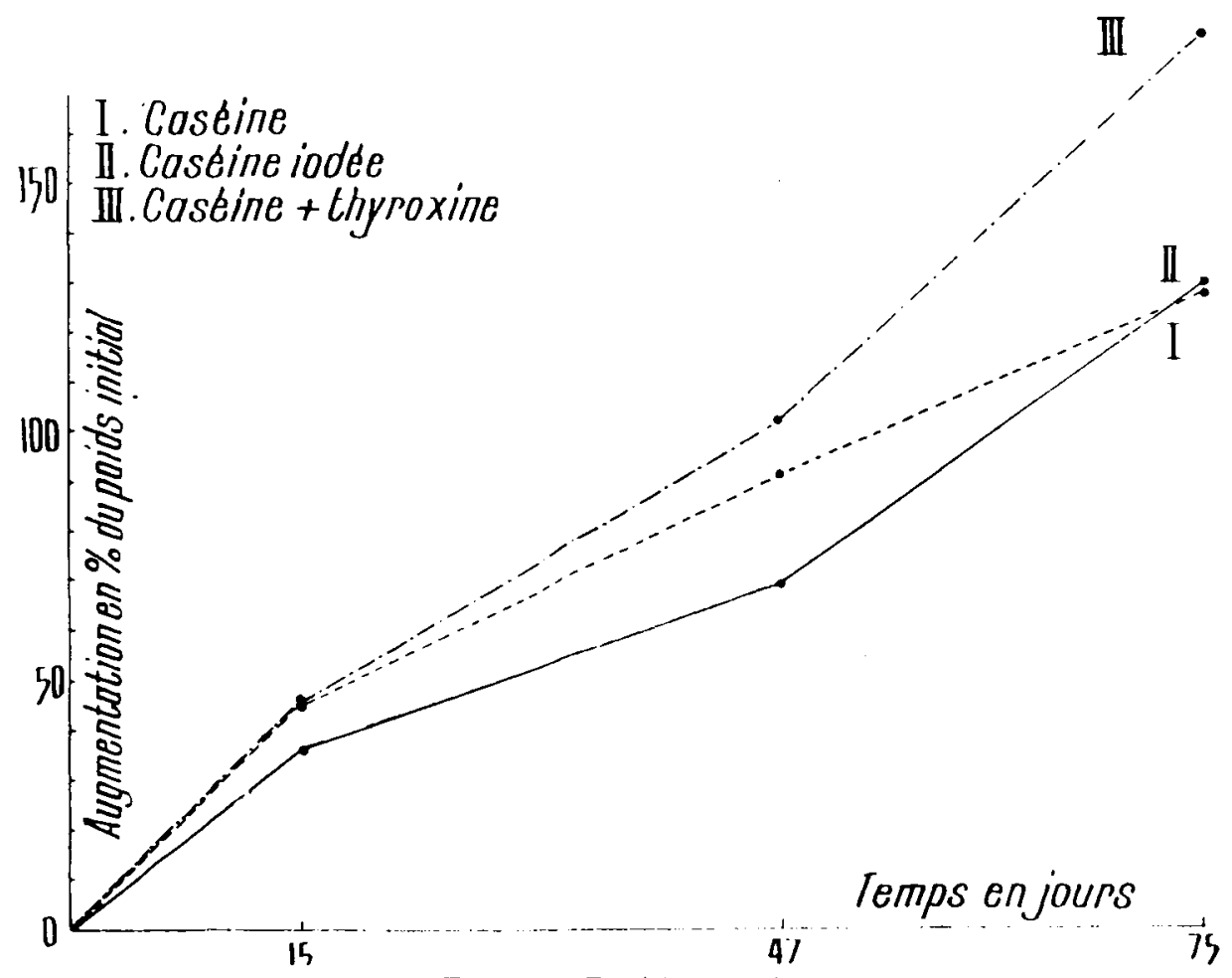

FIG. 1. - Expérience " 1. 
Les régimes II et III ont exercé un effet favorable sur la croissance ; mais alors que cet effet se poursuit dans le lot III pendant toute la durée de l'expérience, il ne se manifeste que pendant les premières semaines dans le lot II.

Il est probable que la teneur en iode élevée du lot II est responsable du freinage de cette accélération initiale de la croissance après quelques semaines de traitement. En effet, le régime II est de dix à vingt fois plus riche en iode que le régime synthétique préconisé pour la Truite par L. E. Wolf (1951), alors que le régime III présente une teneur du même ordre de grandeur que celui-ci.

Cette interprétation est confirmée par les deux expériences suivantes qui portent sur des Truites de poids moyen de 28 grammes et qui ont été poursuivies du 16 Mai au 28 Juin 1953 avec des températures de l'eau ayant varié de $15^{\circ}$ à $21^{\circ}$. Dans ces deux expériences la caséine des lots III était additionnée de thyroxine dans la proportion de $2,4 \%$.

Dans l'expérience $n^{\circ} 2$, les doses quotidiennes de caséine étaient de $50 \mathrm{mg}$. du $16 \mathrm{Mai}$ au 17 Juin, et $60 \mathrm{mg}$. du 17 Juin au 28 Juin.

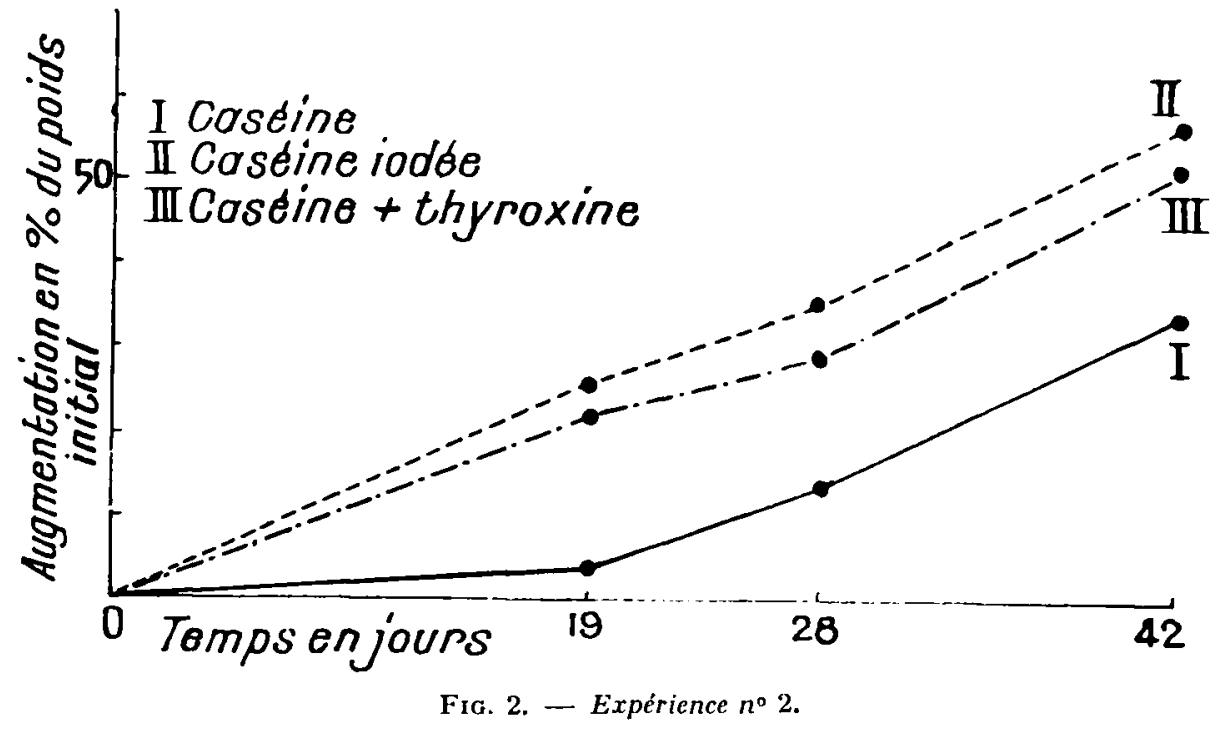

Dans l'expérience $n^{\circ} 3$, ces doses étaient doubles.

Les fig. 2 et 3 illustrent les résultats obtenus.

La croissance des Truites témoins est beaucoup moins satisfaisante dans ces expériences que dans la précédente. Sans doute faut-il incriminer pro parte la saison et la température de l'eau.

L'action nocive de trop fortes teneurs en iode du régime ressort très clairement de la comparaison des expériences 2 et 3 .

Si le fait de doubler la dose de caséine thyroxinée n'a pas entraîné d'inconvénient, cette même opération sur la caséine iodée a été très défavorable et ceci est probablement attribuable à l'augmentation 
beaucoup plus considérable de la teneur en iode du régime qui a entraîné une anorexie très marquée chez la majorité des sujets.

Dans l'expérience 2, les deux régimes iodés ont à peu près la même influence favorable sur la croissance. Si la caséine iodée manifeste une légère supériorité sur le régime thyroxine, ceci doit résulter, d'une part, du fait que l'expérience a été poursuivie moins longtemps que la précédente, d'autre part, du fait que l'écart entre les teneurs en iode des deux régimes est ici moindre puisque la teneur en thyroxine est double de celle de l'expérience précédente. Il est aussi fort possible qu'en cette saison (le printemps), les besoins en iode des jeunes Truites soient supérieurs à ce qu'ils sont en automne et en hiver. Enfin, nous devons peutêtre tenir compte du fait que les Truites de ces deux dernières expériences étaient originaires d'une autre pisciculture que celles de la première

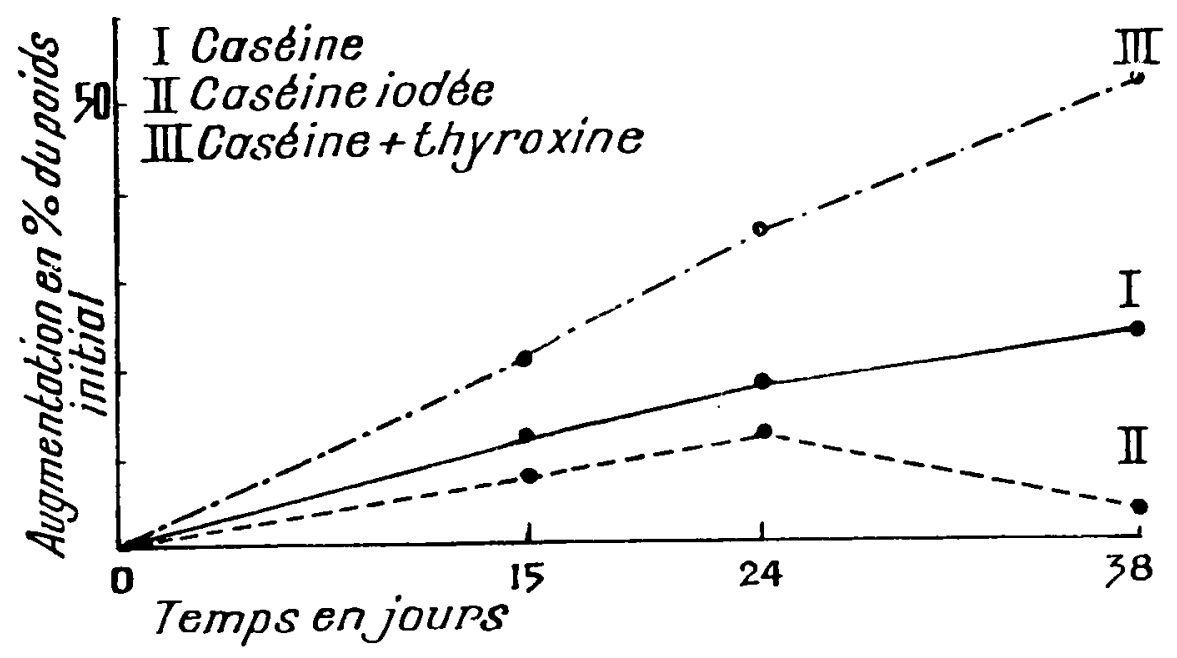

FIG. 3. - Expérience $n^{\circ} 3$.

expérience, bien que la nourriture employée par ces deux piscicultures fut identique (poisson de mer). Ces différences d'origine peuvent prendre une importance plus considérable lorsque les jeunes Truites expérimentées ont reçu au préalable pour régime alimentaire de la viande de boucherie, comme c'est le cas dans une quatrième expérience (faite du 12 Octobre 1953 au $1^{\text {er }}$ Janvier 1954).

Les besoins d'iode de ces Truites peuvent donc être ainsi modifiés, ce qui nous permettrait d'expliquer la différence d'action de la caséine iodée constatée entre cette dernière expérience et la première qui avait été faite à une même saison de l'année et dans des conditions identiques (mêmes doses de caséine iodée, Truites de poids voisin, température de l'eau variant dans des limites très voisines). La seule différence à signaler entre les protocoles des deux expériences concerne les Truites du lot III qui ont reçu ici un mélange caséine + thyroxine à $2,4 \%$, taux double de celui utilisé dans la première expérience.

Dans cette quatrième expérience, les quantités quotidiennes de caséine reçues par chacune des Truites ont été : 
- 40 mg., du 12 Octobre au 9 Novembre;

- $50 \mathrm{mg}$., du 9 Novembre au 8 Décembre;

- 60 mg., du 8 Décembre au 4 Janvier.

La température de l'eau a varié de $7^{\circ}$ à $13^{\circ}$ et le poids moyen des Truites était, avant traitement, de 21 grammes.

La courbe ci-dessous (fig. 4) montre que l'action favorable du

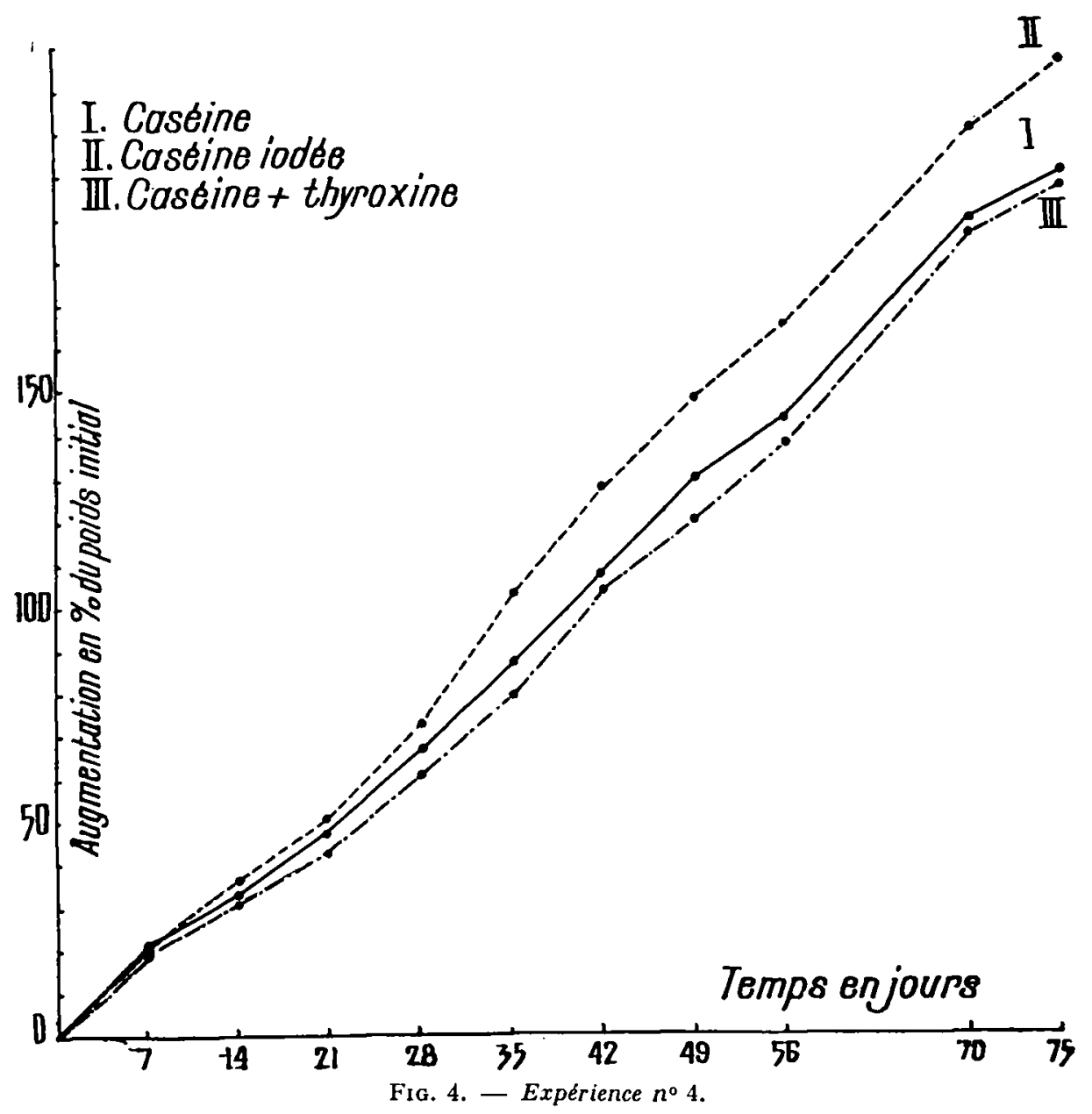

régime II ne se manifeste nettement qu'après quatre semaines de traitement et croît continuellement au cours de l'expérience sans rejoindre la courbe des témoins, comme l'hiver précédent.

Doit-on attribuer cette différence au fait qu'elle s'est déroulée un mois plus tôt que la première dans la saison?

Il est plus probable qu'elle provient de la nature de l'alimentation reçue au préalable par ces Truites à la pisciculture, celles-ci étant sans doute au départ carencées en iode.

D'autre part, le régime III, contrairement à ce que nous attendions 
étant donné les résultats des expériences précédentes, n'exerce pas ici d'action favorable mais plutôt un léger freinage, si toutefois la faible différence enregistrée entre les deux courbes peut être considérée comme significative. Il est vrai que ce taux de $2,4 \%$ de thyroxine n'avait été expérimenté avec succès (expériences II et III) qu'au printemps et ce fait suggère que la posologie efficace de cette hormone peut différer suivant la saison. L'optimum serait moins élevé en automne qu'au printemps où se manifeste chez divers Salmonidés libres une argenture résultant, au moins pro parte, d'une activation thyroïdienne.

Dans toutes ces expériences, les résultats favorables obtenus avec certains régimes alimentaires additionnés de caséine iodée, l'ont été avec des teneurs bien supérieures à celles utilisées avec succès dans l'alimentation du bétail. Nous avons donc expérimenté deux doses de caséine ic dée inférieures.

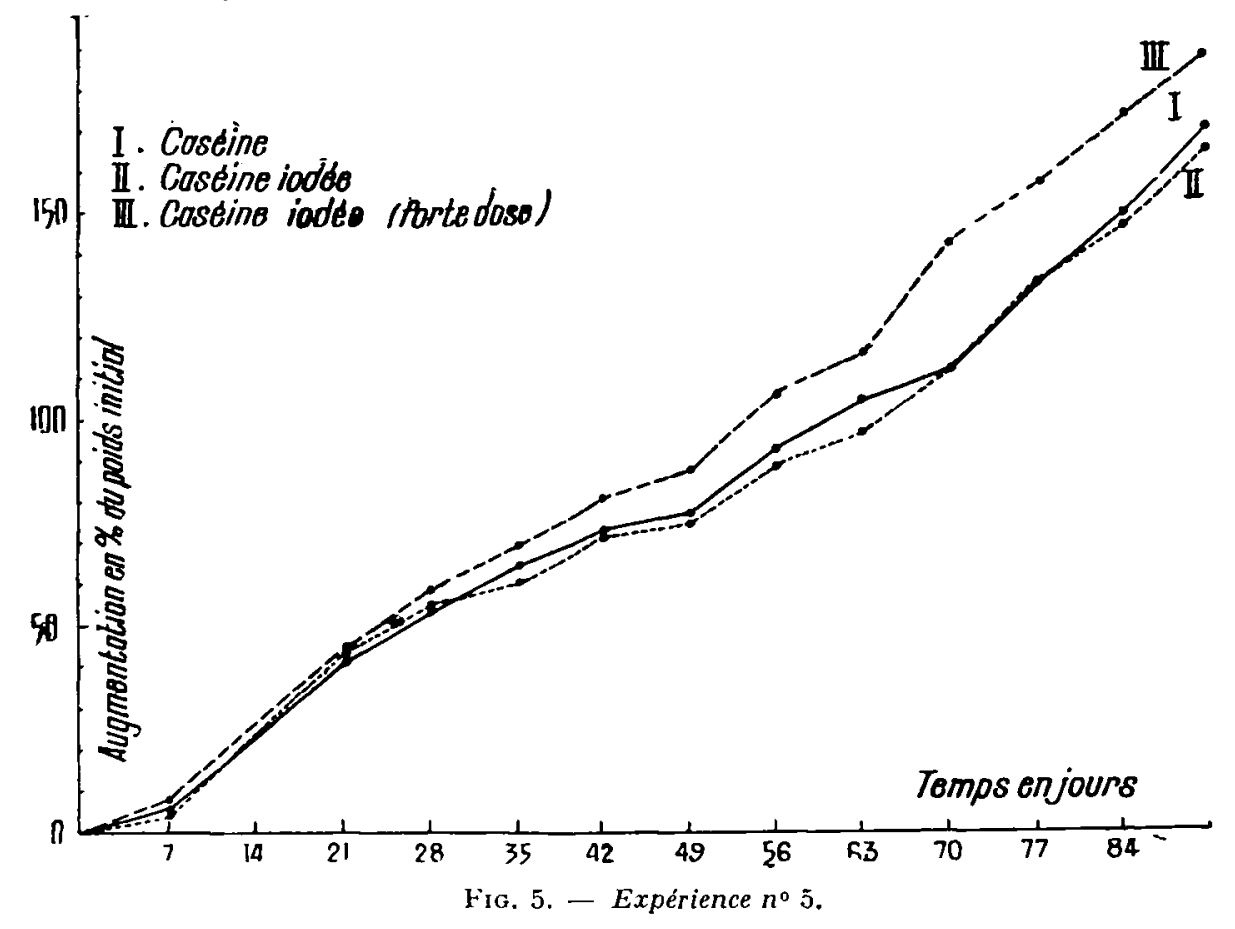

Une cinquième expérience poursuivie du 21 Décembre 1953 au 22 Mars 1954 comprenait trois lots de 19 Truites dont le poids moyen était 34 grammes. La température de l'eau variait à cette époque de $4^{\circ}$ à $12^{\circ}$.

Les Truites, dans cette expérience, ne reçurent pas de thyroxine mais deux lots furent nourris à la caséine iodée. Le lot I, comme précédemment, fut nourri par addition de caséine à l'aliment de base.

Le lot II recevait une dose faible de caséine iodée obtenue en réalisant un mélange caséine + caséine iodée. Jusqu'au 28 Décembre, ce mélange renfermait $1 / 20$ de caséine iodée, puis $1 / 10$ à partir de cette date jusqu'à la fin de l'expérience. 
Le lot III recevait un même poids de caséine iodée pure.

La caséine iodée titrait $1,0 \%$ en thyroxine.

Les doses journalières de caséine furent, par Truite :

- 20 mg., du 21 Décembre au 15 Février;

- 30 mg., du 15 Février au 8 Mars;

- 40 mg., du 8 Mars au 22 Mars.

Ces quantités ayant été augmentées en même temps que le poids de viande ingérée qui a été de 2,3 et 4 grammes par jour, nous avons ainsi réalisé pour le lot III une teneur de $10 \mathrm{mg}$. de caséine iodée par gramme de nourriture et pour le lot II une teneur de $1 \mathrm{mg}$. (sauf pendant les sept premiers jours) cette dernière dose étant utilisée avec succès par les éleveurs de bétail.

On constate, d'après la courbe ci-contre (fig. 5), que cette dose est sans aucun effet tandis que la teneur de $10 \mathrm{mg}$. exerce une action favorable.

\section{Commentaires des résultats obtenus.}

Ces résultats ne sont que des résultats préliminaires, mais ils montrent que, pour certaines doses et au moins à certains stades du développement, l'addition d'iode sous une forme organique plus ou moins proche de la thyroxine, à un régime pauvre en iode, peut accélérer le développement de la Truite arc-en-ciel (1). auteurs.

Nous devons confronter ces résultats à ceux obtenus par d'autres

En 1927 SkLower a étudié l'action d'extrait thyroïdien sur le développement de tout jeunes alevins de Truite.

Il n'a pas observé d'action favorable, mais au contraire une action légèrement défavorable sur la croissance pondérale.

Mais, d'une part, il s'agissait d'une expérience poursuivie sur des alevins très jeunes, au cours des mois qui suivent immédiatement leur éclosion, donc sur des poissons à un stade de développement très différent de celui que nous avons étudié. D'autre part, les renseignements donnés par l'auteur sont insuffisants pour établir une comparaison valable. Nous ignorons notamment la teneur en iode du régime de base (produit Salmona 1), de l'extrait thyroïdien utilisé, de l'activité hormonale de celui-ci, etc.

En 1938, Krockert écrit que l'adjonction de thyroïde à du sang sec n'influence pas la croissance des animaux, mais là encore la discrétion que l'auteur apporte dans la relation de ses expériences ne permet pas d'accorder un bien grand crédit à ses conclusions.

Tout récemment, LA Roche et Leblond ont étudié l'influence des préparations thyroïdiennes et de l'iode sur de jeunes Saumons (Salmo

(i) Nous avons volontairement laissé de côté les modifications morphologiques (argenture, forme de la tête, etc...) sur lesquelles nous reviendrons dans une autre publication. 
salar) et sur Salvelinus fontinalis. Dans les conclusions de ces auteurs, il n'est fait aucune allusion à l'influence de ces préparations sur la croissance, mais, dans la discussion des résultats, le traitement thyroïdien est dit ralentir la croissance pondérale. Toutefois, ne retenant pour les comparer aux nôtres que les expériences d'ingestion de préparations thyroïdiennes, nous constatons ceci :

Dans une expérience, où un extrait commercial thyroïdien était ajouté à du foie de bœuf dans la proportion importante de $50 \%$ du régime, les auteurs observent sur des parrs de 5 à 7 grammes une action défavorable sur la croissance pondérale. Mais il est à peu près certain qu'ils réalisaient dans ce cas une hyperthyroïdisation des poissons et s'ils n'avaient pas effectué deux seules pesées, l'une au début, l'autre à la fin de l'expérience, après 11 semaines de traitement, peut-être auraientils constaté au cours des premières semaines une action favorable?

Par contre dans des expériences où ils additionnaient le mélange foie-rate non plus d'un extrait commercial, mais simplement de thyroïde de bœuf desséchée, substance certainement moins active que la préparation commerciale, LA Roche et LEBLOND obtiennent les résultats suivants :

Dans le tableau 3 donné dans Endocrinology, vol. 51 (1952), page 527, des parrs, suivis pendant dix semaines, de 8 à $11 \mathrm{cms}$. au début de l'expérience, présentent un accroissement de poids hebdomadaire de $0,39 \mathrm{~g}$., alors que les animaux soumis au même régime $+50 \%$ de thyroïde de bœuf desséchée montraient un accroissement de poids de $0,47 \mathrm{~g}$. soit de $20 \%$ supérieur. Dans le tableau 4 (p. 528) les valeurs sont respectivement de 0,15 g. et 0,23 g., soit de $53 \%$ supérieur chez les animaux traités par le régime contenant de la thyroïde, pour des parrs de 5 à $15 \mathrm{cms}$. Il nous semble donc qu'il existe une action incontestable de la thyroïde desséchée sur la croissance du parr. Il est vrai que, dans les dites expériences, aucune action ne paraît s'exercer sur le frai (poissons au-dessous de $5 \mathrm{cms}$.) ni sur le smolt.

Mais peut-être ce résultat signifie-t-il que les doses actives devraient être différentes à ces stades (1)? En ce qui concerne le smolt, ne nous étonnons pas de cette différence de sensibilité aux préparations thyroïdiennes, puisque son fonctionnement thyroïdien est nettement différent de celui du parr (Fontaine, Leloup, Olivereau) et son iodémie est, notamment, beaucoup plus élevée (2).

Quoi qu'il en soit, de l'ensemble des résultats actuellement obtenus, on peut conclure qu'au moins à un certain état du développement, l'addition de préparations iodées organiques, thyroxine ou substances iodées

(1) Il nous paraît intéressant de rapprocher ces résultats de LA Roche et LEBLOND, action très différente des préparations thyroïdiennes selon le stade, de ceux de Smith et Matrhews selon lesquels l'injection d'extraits de glande thyroïde de poisson perroquet à des grogneurs (Hoemulon $S p$.) ne détermine une élévation du métabolisme respiratoire que sur des poissons de 15 à 32 grammes. douce.

(2) Bien que les représentants de ces deux stades aient été capturés en eau 
d'activité physiologique à des régimes alimentaires, peut entraîner une accélération de la croissance pondérale des Salmonidés.

Si l'emploi des préparations thyroïdiennes semble peu susceptible d'ètre appliqué sur une grande échelle, il n'en est pas de même des caséines iodées dont nous montrons ici l'efficacité. On sait d'ailleurs qu'une riche expérimentation de ces substances a été faite ces dernières années sur le bétail et l'on en trouvera l'analyse dans l'excellent rapport de M. Brunaud. La plupart des auteurs qui ont essayé de nombreuses doses de caséine iodée (notamment R. Braude dans ses multiples expériences sur les porcs) concluent que les doses fortes ont un effet défavorable sur la croissance tandis que les faibles doses provoqueraient, en général, un léger accroissement de poids. Et la grande diversité des résultats obtenus semble bien tenir au fait que certains auteurs n'ont pas étudié une gamme de doses assez étendue et ceci à des étapes variées du développement de l'animal. Or, il est certain que les besoins de l'organisme en hormones thyroïdiennes ou en corps voisins varient selon le stade du développement. Il est évident aussi que la création d'un état de forte hyperthyroïdie est néfaste du fait que l'accroissement du métabolisme entraîne une consommation exagérée d'aliments et cependant un amaigrissement des animaux, et c'est sans doute le cas des expériences ayant abouti à un résultat défavorable.

En zootechnie donc, aussi bien dans l'élevage du bétail qu'en pisciculture, une expérimentation méthodique et patiente doit préciser la dose optima de substance active pour un état de développement déterminé. C'est dans ce sens que nous poursuivons nos recherches sur les Salmonidés.

\section{RÉsumé.}

La teneur en protéines iodées du régime alimentaire de la Truite arc-en-ciel intervient de façon notable dans la croissance de cette espèce. Il semble exister une dose optima qui varie sans doute avec l'âge, l'état physiologique du poisson, la durée d'administration du régime, la saison, etc.

Dans les conditions expérimentales ci-dessus précisées, et notamment avec une alimentation de base (muscle de mammifère) pauvre en iode, la dose favorable de caséine iodée ajoutée au régime était comprise entre 10 et $50 \mathrm{mg}$. par jour pour des Truites de 20 à 65 grammes. 


\section{BIBLIOGR A PHIE}

Braude (R.). - The effect of feeding iodinated casein to pigs. J. Agr. Sc., 1947, 37-45.

Brunaud (M.). - Facteurs physiologiques internes de la production de la viande (action des glandes endocrines). Annales de la nutrition et de l'alimentation, 1952 , vol. $6, \mathrm{n}^{\text {os }} 4-5$, pp. 195-221.

Fontaine (M.), Leloup (J.) et Olivereau (M.). - La fonction thyroïdienne du jeune Saumon, Salmo salar L. (parr et smolt) et son intervention possible dans la migration d'avalaison. Arch. Sc. Physiol., 1952, t. 6, pp. 83-104.

Krockert (G.). - Herstellung und Prüfung eines Trockenfütters unter Berücksichtigung von Vitaminen, Mineralstoffen, und Hormonen zur Aufzucht und Mast von Forellen. Zeitschrift für Fischerei, 1938, t. 36, pp. 473-487.

LA Roche (G.) et Leblond (C. P.). - Effect of thyroïd preparations and iodide on Salmonidae. Endocrinology, vol. 51, n 6, 1952, pp. 524-545.

Randoin (L.). - Tables de composition des aliments. Lanore, Paris, 1947.

SkLower. - Uber den Einfluss von Schildrüsen und Thymus Fütterung auf die Körperlänge und das Gewicht von Forellenbrut. Zeitschrift für Fischerei, 1927, vol. XXV, pp. 549-553.

Sмrth (D. C.) et Matthews (S.). - The effect of extracts of the thyroid gland of the Bermuda parrot fish on the oxygen consumption of Bermuda white grunts (Hoemulon Sp.). Anat. Rec., 1947, vol. 99, n 4, p. 593.

Wolf (L. E.). - Diet experiments with Trout. The progressive fish-culturist, Janv. 1951, pp. 17-24. 